\title{
FACING THE CHALLENGE OF A FUNCTIONAL CHARACTERIZATION OF TOLL-LIKE RECEPTOR (TLR)1 AND TLR2 IN COMMON CARP
}

\author{
Inge R Fink, Maria Forlenza, Danilo Pietretti, Geert F Wiegertjes* \\ Cell Biology and Immunology group, Department of Animal Sciences, Wageningen University, \\ Wageningen $6700 \mathrm{AH}$, The Netherlands
}

\author{
*Corresponding author. Tel.: +31 317482732 \\ E-mail address: geert.wiegertjes@wur.nl
}

\begin{abstract}
Toll-like receptors are a family of germline-encoded pattern recognition receptors which activate rapid inflammatory responses upon detection of their cognate ligands. In mammals, TLR2 can form a heterodimer with TLR1, 6, or 10, and recognize lipoproteins/lipopeptides from Gram-positive bacteria. Of these TLRs, only $t l r l$ and $t l r 2$ are present in fish genomes. In the carp genome, a single full-length $t / r 1$ gene could be identified, whereas two functional genes for carp $t l r 2$ exist.

High expression of $t l r 1$ and both $t l r 2$ genes was found in immune organs and leukocytes of both myeloid and lymphoid origin. Of the two $t l r 2$ genes, $\operatorname{tl} 2 a$ was always higher expressed than $t l r 2 b$. Three-dimensional modelling of the carp Tlr 1 and Tlr2 proteins appear to confirm the ability to form a heterodimer, with a pocket that can accommodate the tri-acylated lipopeptide ligand Pam3CSK4, similar to the heterodimer structure of human TLR1-TLR2 on which the model was built. In cell lines of human as well as fish origin transfected to overexpress the carp Tlr proteins, co-localization of carp Tlr1 and Tlr2 could be revealed with confocal microscopy. We were unable to confirm Tlr localization to the cell surface, the expected sub-cellular localization of Tlr1 and Tlr2. Further experimental evidence would be needed to unequivocally prove molecular interaction between the two Tlr proteins.

In previous work, we have described ligand-specific activation of carp Tlr2 overexpressed in human cells (HEK), via measurement of increased phosphorylation levels of the MAP kinase p38 by Western blot. Instead of this semi-quantitative method, here we used a read-out system based on NF-kB activation and subsequent measurement of luminescence. This quantitative method could not confirm our initial ligand binding studies, not using human cell lines (HEK, HeLa), nor using a fish cell line (EPC). We will discuss possible explanations for the unsuccessful ligand-specific activation of NF-kB after overexpression of Tlr1 and/or Tlr2 in human, but also fish cell lines, to propose alternative future strategies for studying ligand binding properties of fish Tlrs.
\end{abstract}

\section{KEYWORDS}

Carp; TLR1; TLR2, sub-cellular localization, ligand binding 